\title{
Fundamental Study on Development of Polymer Nano-Film Synthesized on Self-Assembled Phospholipid Layer Fabricated by Plasma-Assisted Method
}

\author{
Shin-ichi Kondo ${ }^{1 *}$, Yasushi Sasai ${ }^{1}$, Naoki Doi ${ }^{1}$, Yukinori Yamauchi ${ }^{2}$, \\ and Masayuki Kuzuya ${ }^{3}$ \\ ${ }^{1}$ Laboratory of Pharmaceutical Physical Chemistry, Gifu Pharmaceutical University, \\ 1-25-4 Daigaku-Nishi, Gifu 501-1196, Japan \\ ${ }^{2}$ Department of Pharmaceutical Physical Chemistry, College of Pharmaceutical Sciences, \\ Matsuyama University, 4-2 Bunkyo-cho, Matsuyama, Ehime 790-8578, Japan \\ ${ }^{3}$ Department of Health and Welfare, Faculty of Human Welfare, Chubu Gakuin \\ University, 2-1 Kirigaoka, Seki-shi, Gifu 501-3993, Japan \\ *skondo@gifu-pu.ac.jp
}

\begin{abstract}
The morphology of polymer nano-film in organic solvents and water was studied by ${ }^{1} \mathrm{H}-\mathrm{NMR}$ and atomic force microscope (AFM) measurement. $\quad{ }^{1} \mathrm{H}-\mathrm{NMR}$ spectra of polymer nano-film in $\mathrm{CD}_{3} \mathrm{OD}$ suggested that the crosslinking and stearic acid moiety could freely move. It was also shown that the morphology of polymer nano-film in $\mathrm{CDCl}_{3}$ might be cylindrical or spherical structure with stearic acid moiety as outer shell, and that in $\mathrm{D}_{2} \mathrm{O}$ the stearic acid moiety might exist in the inner core. It was also indicated from AFM images that polymer nano-film kept a spread form in methanol and a particle-like shape in chloroform and water.
\end{abstract}

Keywords: Polymer nano-film, Self-assembled phospholipid layer, Plasma irradiation, Cyclodextrin

\section{Introduction}

The nano-film is a new type of material that possesses a two-dimensional polymeric structure with nano-meter thickness. Recently, nano-films fabricated from molecular, atomic, and ionic components have been extensively investigated for systems with analytical and biomedical applications, such as separation matrices and drug delivery carriers [1-5].

We have reported a novel method to introduce a durable surface wettability and minimize its decay with time on several hydrophobic polymers, such as polyethylenenaphthalate, polyethylene, and nylon-12 by plasma-assisted method [6-10]. By exploiting the durable hydrophilic surface property, we have also developed a novel fabrication of self-assembled phospholipid (phosphatidyl choline (PC)) layer on hydrophobic polymer surface [11]. The self-assembled phospholipid layer
(LDPE-PC-SA) was thermally stable up to $80{ }^{\circ} \mathrm{C}$. By the introduction of stearic acid (StA) into LDPE-PC-SA, enzyme, antibody or cyclodextrin derivatives could be immobilized onto the LDPE-PC-SA. It was also shown that the LDPE-PC-SA possessed fluidity.

In the previous paper we fabricated the polymer nano-film possessing hydrophilic and hydrophobic surface with LDPE-PC-SA containing StA [12]. Figure 1 shows the schematic illustration of fabrication of polymer nano-film. Per-6-amino$\beta$-cyclodextrin (Per-6-ABCD) was immobilized onto the LDPE-PC-SA containing StA, and then crosslinked with 4,4'-[1,2-ethanediyl-di-(oxyethylamino)]-bis(4-oxo-2-butenoic acid) (EBBA) to fabricate the polymer nano-film. The dynamic light scattering (DLS) measurement indicated that the morphology of polymer nano-film in aqueous solution could be changed by $\mathrm{pH}$, and that the 
polymer nano-film can form a spherical structure in acid condition.

In this communication, we further studied the morphology of polymer nano-film in organic solvents (methanol and chloroform) and water. ${ }^{1} \mathrm{H}-\mathrm{NMR}$ spectra measurement of polymer nano-film were performed to obtain the insight on the mobility of crosslinking part and alkyl chain of stearic acid moiety. The morphology of polymer nano-film was also estimated by atomic force microscope (AFM) measurement.

\section{Experimental}

\subsection{Materials}

Per-6-amino- $\beta$-cyclodextrin (Per-6-ABCD) and 4,4'-[1,2-ethanediyl-di(oxyethylamino)]-bis-(4oxo-2-butenoic acid) (EBBA) were synthesized according to the literatures $[12,13]$.

\subsection{Fabrication of self-assembled phospholipid} layer

The LDPE-VEMAC film was prepared according to the method reported previously [11]. Hexamethylene diamine (HMDA) was introduced into LDPE-VEMAC film by the condensation reaction to obtain the LDPE film grafting HMDA, LDPE-HE. The self-assembled PC layer incorporating stearic acid (StA) (LDPE-StA-PCSA) was fabricated with phosphatidyl choline (PC) suspension containing StA (PC:StA=1:6) $(10 \mathrm{~mL})$ at $30^{\circ} \mathrm{C}$. The film was washed with water, and dried in vacuo.

2.3. Fabrication of polymer nano-film on
LDPE-StA-PC-SA film immobilizing Per-6-ABCD

The polymer nano-film was prepared according to the literature [12]. To $5 \mathrm{~mL}$ of $0.016 \mathrm{nmol} / \mathrm{mL}$ EBBA solution was added $4.8 \mathrm{mg}$ of EDC-HCl. This solution was kept at $30{ }^{\circ} \mathrm{C}$ for $2 \mathrm{~h}$. The LDPE-StA-PC-SA film immobilizing Per-6-ABCD was soaked into this solution at $30^{\circ} \mathrm{C}$ for $72 \mathrm{~h}$. This film was washed with PBS. The film was immersed into $5 \mathrm{~mL}$ of ethanol and kept at $40{ }^{\circ} \mathrm{C}$ for $12 \mathrm{~h}$. This ethanol solution was transferred through a $0.45 \mu \mathrm{m}$ filter (GL Chromatodisc $13 \mathrm{~N}$, GL Sciences Inc.) into a pre-swollen semi-permeable membrane (Spectra/Por ${ }^{\circledR} 2$ Dialysis Membrane Standard RC Tubing MWCO: 12-14,000, Spectrum Laboratories, Inc.; molecular weight cutoff, $12,000-14,000 \mathrm{~g} / \mathrm{mol})$. The both sides of the tube were sealed with dialysis tubing closures (Dialysis Tubing Closures Standard Closure Type, $35 \mathrm{~mm}$ ). The solution was dialyzed against $200 \mathrm{~mL}$ of methanol for $24 \mathrm{~h}$ and then against $200 \mathrm{~mL}$ of distilled water for $24 \mathrm{~h}$.

\subsection{Atomic force microscope measurement in} dynamic force mode

The morphology of polymer nano-film was investigated by atomic force microscopy (AFM) with dynamic force mode employing Scanning Probe Microscopy AFM-5400L (Hitachi High-Technologies Co.) and cantilever (MPP-11100-10, Veeco). The solution $\left(\mathrm{CH}_{3} \mathrm{OH}\right.$, $\mathrm{CHCl}_{3}$ and $\mathrm{H}_{2} \mathrm{O}$ ) of polymer nano-film was casted on mica, and then dried in vacuo to obtain AFM sample. AFM measurement was performed under atmospheric conditions.

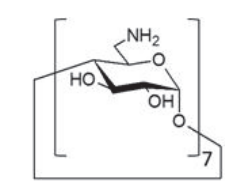

Per-6-amino- $\beta$-cyclodextrin (Per-6-ABCD)

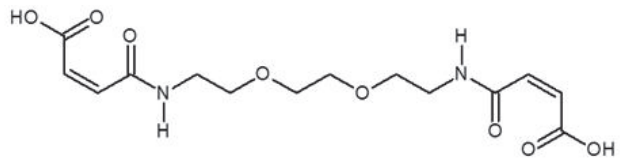

4,4'-[1,2-ethanediyldi(oxy-ethylamino)] bis-[4-oxo-2-butenoic acid] (EBBA)
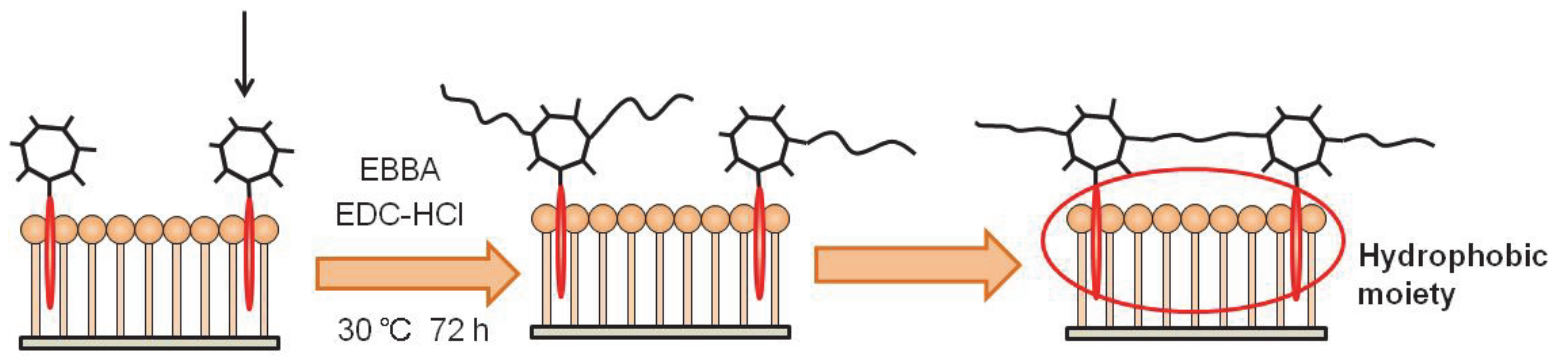

Fig. 1. Schematic illustration of the fabrication of polymer nano-film possessing hydrophilic and hydrophobic side with the self-assembled phospholipid layer containing stearic acid. 


\section{Results and discussion}

\section{1. ${ }^{1} \mathrm{H}-\mathrm{NMR}$ spectra of polymer nano-film}

Figure 2 shows ${ }^{1} \mathrm{H}-\mathrm{NMR}$ spectra of polymer nano-film in $\mathrm{CD}_{3} \mathrm{OD}, \mathrm{CDCl}_{3}$ and $\mathrm{D}_{2} \mathrm{O}$. The peaks around 0.9 and between 1.0 and $1.2 \mathrm{ppm}$ were ascribed to the methyl and methylene groups of stearic acid moiety, respectively. The peaks of methylene groups of EBBA were observed between 1.9 and $2.2 \mathrm{ppm}$. The methine and methylene protons of Per-6-ABCD were also observed between 4.0 and 4.2 , and about $5.2 \mathrm{ppm}$ with weak intensity, respectively. It was considered that the broadening of peaks might be depended on the mobility of the moiety. The peaks of methyl and methylene group of stearic acid moiety in $\mathrm{D}_{2} \mathrm{O}$ were broader than $\mathrm{CD}_{3} \mathrm{OD}$ and $\mathrm{CDCl}_{3}$, indicating that the mobility of stearic acid moiety could be restricted. This result suggested that the stearic acid moiety of polymer nano-film might exist in the inner core in $\mathrm{D}_{2} \mathrm{O}$ as expected, and that the stearic acid moiety in organic solvent might be exposed to a solvent.

On the other hand, the peaks of methylene groups of EBBA in $\mathrm{CDCl}_{3}$ were broader than $\mathrm{CD}_{3} \mathrm{OD}$, so that the mobility of crosslinking part in
$\mathrm{CDCl}_{3}$ could be suppressed compared to that in $\mathrm{CD}_{3} \mathrm{OD}$. It was assumed that the crosslinking part of polymer nano-film could freely move in $\mathrm{CD}_{3} \mathrm{OD}$ and that the morphology of polymer nano-film in $\mathrm{CDCl}_{3}$ might be cylindrical or spherical structure with stearic acid moiety as outer shell.

3.2. Morphology estimation of polymer nano-film by AFM measurement

In order to gain the insight on the morphology of polymer nano-film, AFM measurement was performed. Figure 3 shows AFM image of polymer nano-film.

The sample prepared from methanol solution of polymer nano-film showed a sheet-like structure, about $150-200 \mathrm{~nm}$ on a side (Fig. 3 (A)). On the other hand, the sample prepared from chloroform and water demonstrated a particle-like object, about $50-100 \mathrm{~nm}$ in diameter (Figs. 3(B) and (C)). These results of AFM images were correlated to those of ${ }^{1} \mathrm{H}-\mathrm{NMR}$ spectrum. In other words, polymer nano-film kept a spread form in methanol and a particle-like shape in chloroform and water.

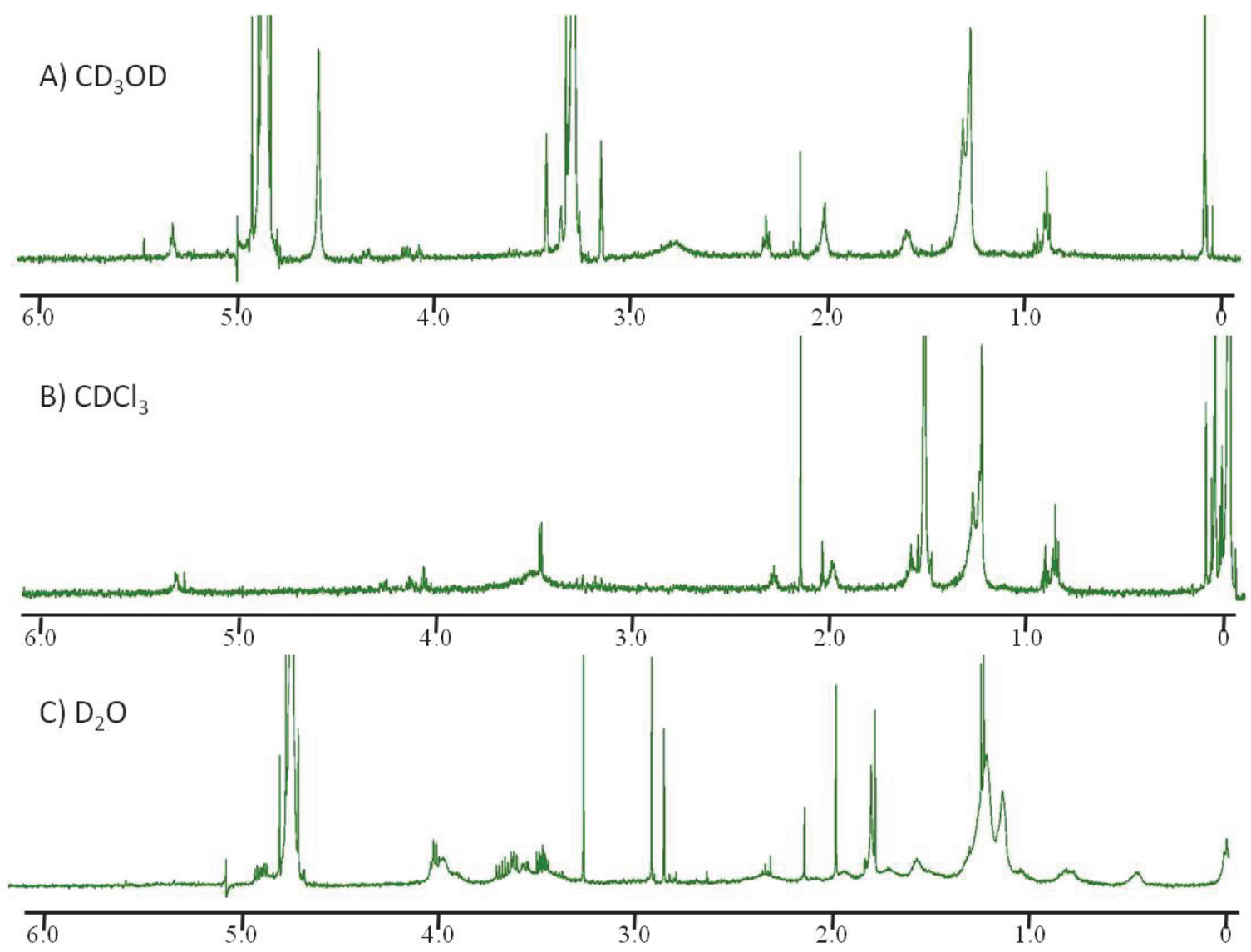

Fig. 2. ${ }^{1} \mathrm{H}-\mathrm{NMR}$ spectra of polymer nano-film in $\mathrm{CD}_{3} \mathrm{OD}(\mathrm{A}), \mathrm{CDCl}_{3}(\mathrm{~B})$, and $\mathrm{D}_{2} \mathrm{O}(\mathrm{C})$. 
A) $\mathrm{CH}_{3} \mathrm{OH}$

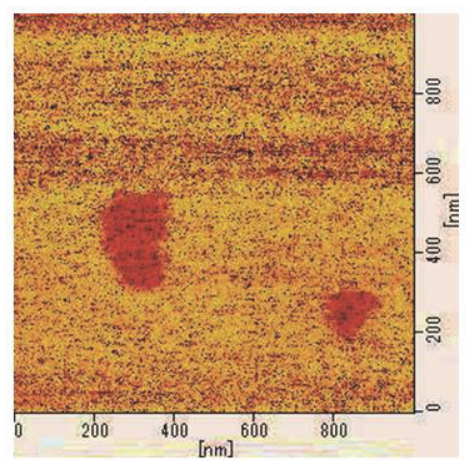

B) $\mathrm{CHCl}_{3}$

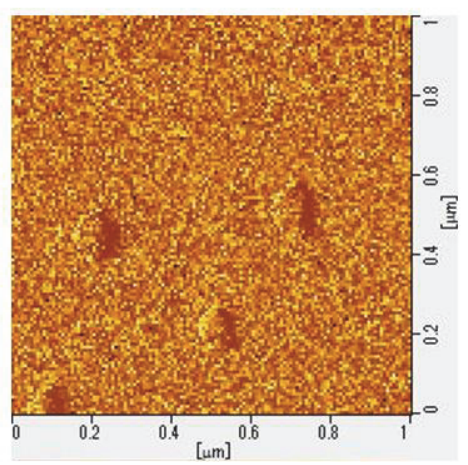

C) $\mathrm{H}_{2} \mathrm{O}$

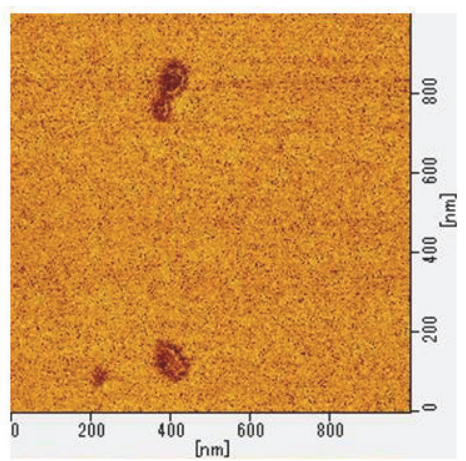

Fig. 3. AFM images of polymer nano-film.

\section{Conclusion}

The conclusions drawn from the present study can be summarized as follows.

It was suggested from ${ }^{1} \mathrm{H}-\mathrm{NMR}$ spectra that the crosslinking and stearic acid moiety in polymer nano-film could freely move in $\mathrm{CD}_{3} \mathrm{OD}$, that the morphology in $\mathrm{CDCl}_{3}$ might be cylindrical or spherical structure with stearic acid moiety as outer shell, and that the stearic acid moiety of polymer nano-film might exist in the inner core in $\mathrm{D}_{2} \mathrm{O}$. It was also indicated from AFM images that polymer nano-film kept a spread form in methanol and a particle-like shape in chloroform and water. The results of AFM images were correlated to those of ${ }^{1} \mathrm{H}-\mathrm{NMR}$ spectra.

We are now actively elaborating the control of the size of polymer nano-film and aiming to the application of drug delivery system as a novel carrier.

\section{References}

1. T. Bauer, Z. Zheng, A. Renn, R. Enning, A. Stemmer, J. Sakamoto, and A. D. Schlüter, Angew. Chem., 50 (2011) 7879.

2. S. Motoyama, R. Makiura, O. Sakata, and H. Kitagawa, J. Am. Chem. Soc., 133 (2011) 5640.

3. Z. Zheng, L. Opilik, F. Schiffmann, W. Liu, G. Bergamini, P. Ceroni, L.-T. Lee, A. Schlütz, J.
Sakamoto, R. Zenobi, J. VandeBondele, and A. D. Schlüter, J. Am. Chem. Soc., 136 (2014) 6103.

4. K. Terada, R. Sakamoto, S.-T. Yi, S. Katagiri, T. Kambe, and H. Nishihara, J. Am. Chem. Soc., 137 (2015) 4681.

5. N. Idota, S. Fukuda, T. Tsukahara, and Y. Sugahara, Chem. Lett., 44 (2015) 203.

6. M. Kuzuya and T. Yamashiro, J. Photopolym. Sci. Technol., 8 (1995) 381.

7. M. Kuzuya, Y. Matsuno, T. Yamashiro, and M. Tuiki, Plasmas Polym., 2 (1997) 79.

8. M. Kuzuya, T. Yamashiro, S. Kondo, and M. Tuiki, Plasmas Polym., 2 (1997) 133.

9. M. Kuzuya, T. Sawa, T. Yamashiro, S. Kondo, and O. Takai, J. Photopolym. Sci. Technol., 14 (2001) 87.

10. S. Kondo, Y. Sasai, and M. Kuzuya, Thin Solid Films, 515 (2007) 4136.

11. S. Kondo, Y. Sasai, Y. Yamauchi, and M. Kuzuya, J. Photopolym. Sci. Technol., 22 (2009) 477.

12. S. Kondo, T. Kusumoto, Y. Sasai, N. Doi, Y. Yamauchi, and M. Kuzuya, J. Photopolym. Sci. Technol., 29 (2016) 439.

13. S. Kondo, M. Suzuki, Y. Sasai, Y. Yamauchi, and M. Kuzuya, J. Photopolym. Sci. Technol., 26 (2013) 545. 